\title{
Belphégor
}

\section{Nicolas Rouvière (dir.), Bande dessinée et enseignement des humanités}

\section{Vittorio Frigerio}

\section{(2) OpenEdition}

1 Journals

\section{Édition électronique}

URL : http://journals.openedition.org/belphegor/504

DOI : 10.4000/belphegor.504

ISSN : 1499-7185

Éditeur

LPCM

\section{Référence électronique}

Vittorio Frigerio, «Nicolas Rouvière (dir.), Bande dessinée et enseignement des humanités », Belphégor [En ligne], 12-1 | 2014, mis en ligne le 12 juin 2014, consulté le 22 septembre 2020. URL : http:// journals.openedition.org/belphegor/504; DOI : https://doi.org/10.4000/belphegor.504

Ce document a été généré automatiquement le 22 septembre 2020.

\section{(c) (i) (9)}

Belphégor est mis à disposition selon les termes de la Licence Creative Commons Attribution - Pas d'Utilisation Commerciale - Pas de Modification 4.0 International. 
Nicolas Rouvière (dir.), Bande dessinée et enseignement des humanités

\author{
Vittorio Frigerio
}

\title{
RÉFÉRENCE
}

Rouvière Nicolas (dir.), Bande dessinée et enseignement des humanités. Sous la direction de. Grenoble : ELLUG, 2012. 434 p. ISBN : 978-2-84310-225-7 
Déjà en 1955, Le Fureteur, dans sa rubrique hebdomadaire dans le journal de Spirou, répondait aux lecteurs qui se plaignaient en disant «Mon professeur ne veut pas qu'on lise des illustrés » en leur conseillant: "Faites voir Spirou à vos parents. À vos professeurs. Montrez-leur les dessins pleins de goût, les articles instructifs, les histoires délicieuses que renferme votre journal. Un simple coup d'œil leur suffira pour qu'ils se rendent compte que Spirou est un hebdomadaire de classe, bien au-dessus de ces vilains petits journaux mal réalisés ${ }^{1}$ ». Près de soixante ans après, l'acceptation graduelle de la bande dessinée (hebdomadaires « de classe » et autres) dans la grande famille de la littérature exige que l'on se pose aussi un certain nombre de questions sur son insertion dans les écoles, le rôle

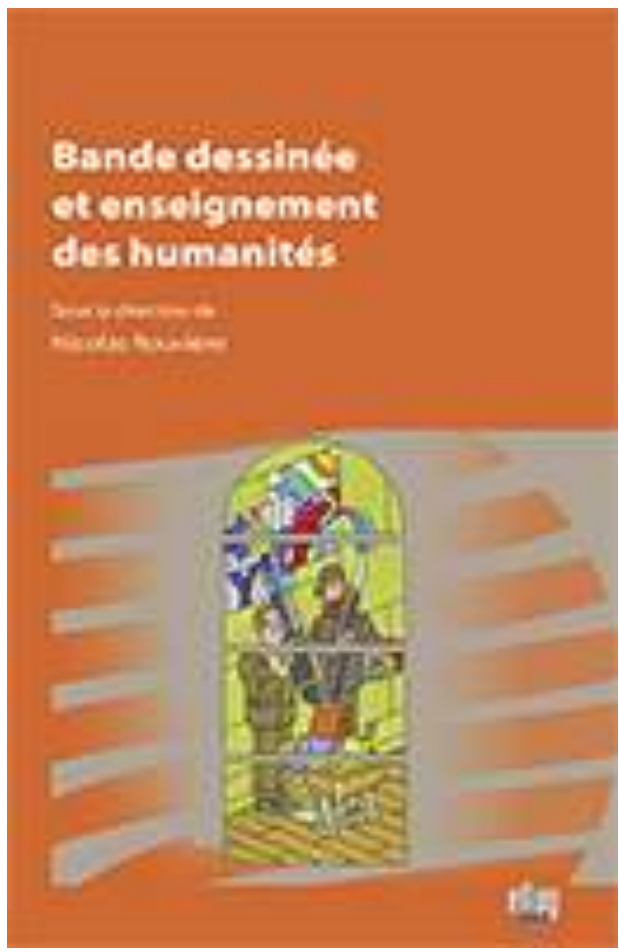
qu'elle peut y jouer et les usages auxquels on la soumet. Cet ouvrage, qui doit sa naissance au colloque « Lire et produire des bandes dessinées à l'École ", tenu en 2010 à l'université Grenoble 3, propose un vaste éventail de réflexions sur le thème, fournissant pour la bande dessinée franco-belge un cadre critique de référence qui n'est pas dissemblable de celui fourni par Stephen Tabachnick pour les comics anglo-saxons dans son ouvrage Teaching the Graphic Novel, avec l'intention de fournir une «réflexion pédagogique et didactique sur l'usage scolaire du médium » (13), surtout dans les classes de littérature, d'histoire, de langue et de civilisation, et ce principalement au niveau de l'école primaire, du collège et du lycée.

Divisé en quatre sections («De la BD-prétexte à l'étude du médium pour lui-même : enjeux pédagogiques et obstacles culturels", "La bande dessinée en classe de littérature : quel projet éducatif et quelle didactique pour interroger les œuvres?", «Bande dessinée et enseignement de l'histoire: quel usage pour quels apprentissages ? ", "L'étude la bande dessinée en cours de langue et de civilisation »), l'ouvrage offre tout autant des considérations d'ordre théorique et général sur le thème que des études de cas particuliers. On ne peut ici faire pleinement justice à chaque intervention, mais relevons l'intérêt de l'étude de Bernard Tabuce sur la place de la bande dessinée dans les manuels de collège. Harry Morgan, quant à lui, identifie des constantes tout aussi frappantes qu'inquiétantes dans l'approche à la bande dessinée préconisée par les «professionnels de l'enfance» (58) tout au long de l'existence du médium, des années trente à l'époque contemporaine, en passant par le règne de la Commission de surveillance dans les années 1950 et 1960. La "persistance d'attitudes anciennes " (70) se révèle aussi à travers l'insistance à vouloir "éduquer pour édifier » (71), même si le signe politique sous lequel on s'adonne à cette activité peut maintenant être inversé par rapport au passé. Nicolas Rouvière examine l'utilisation de bandes dessinées en classe au cycle 3, passant en revue diverses approches utiles (dissociation 
des composantes textuelle et iconique, fragmentation, abstraction d'éléments porteur de sens pour faire ressortir l'effet narratif et plastique, ou encore soulignement des analogies, mise en réseau avec d'autres supports, etc.). Jean-Paul Meyer se penche sur l'adaptation de romans en bande dessinée, montrant l'évolution d'une conception proche de la simple illustration à de nouveaux types d'adaptation qui arrivent à sauvegarder les spécificités uniques du récit en image. Une thématique voisine est explorée par Guillaume Perrier, qui disserte de plusieurs tentatives d'adaptation de Proust en bande dessinée pour conclure à l'utilisation possible de celle-ci en «supplément» à l'œuvre, éventuellement pour en saisir quelques images « mémorables » qui pourront « vivre dans l'esprit des jeunes lecteurs» (197). Une autre proposition d'utilisation "partielle» de la bande dessinée est proposée par Thierry Crépin dans son parcours au travers de la propagande dans les illustrés pour la jeunesse sous l'Occupation. En classe d'histoire, la bande dessinée fait alors fonction de « source documentaire iconographique au même titre que la photographie ou l'affiche », plutôt que d'être «exploitée [...] comme un média spécifique » (256). C'est dans la perspective de l'Histoire culturelle que Joël Mak dit Mack aborde l'usage de la bande dessinée en classe comme document historique. Il identifie « deux axes d'étude en classe : l'examen critique de la dimension documentaire présente dans les fictions historiques [...] et l'étude de la bande dessinée comme document sur les représentations de son temps " (272), pour se pencher ensuite sur le cas de la bande dessinée-reportage. La BD, pour lui, serait tout autant un véritable objet historique » qu'un " matériau particulièrement efficace pour s'interroger [...] sur ce qu'est un document historique » (280).

Une réflexion sur le rôle de la bande dessinée à l'université, dans une classe de langue, nous est fournie par Jean-Pierre Thomas dans sa contribution, "Enseigner la bande dessiné à Toronto: questions de tradition », et une autre par Marc Blancher, qui apporte un témoignage réfléchi sur deux expériences d'enseignement d'Astérix en contexte germanophone (l'une au niveau du collège en Suisse alémanique, l'autre au niveau universitaire en Allemagne), relevant comment ce genre d'entreprise peut souvent donner des résultas pédagogiques mitigés et « occasionn[er] chez l'enseignant un sentiment d'insatisfaction » (301), que partageront tous ceux qui se sont essayés au même exercice en classe de français langue seconde. D'autres aires linguistiques sont explorées par Sylvie Martin-Mercier, qui consacre son article aux histoires du Commissaire Spada, dessiné par Gianni De Luca, un des plus grands fumettisti italiens, offrant des suggestions très concrètes et multiples pour l'utilisation de ces œuvres en salle de classe, et par Tatiana Blanco-Cordon, qui fait de même pour l'enseignement de l'espagnol langue seconde, à l'aide d'un récit de bande dessinée de l'auteur Alfonso López.

4 Ce recueil vient à point nommé, avec son intention de défendre la place de la bande dessinée dans les humanités - pour faire en sorte qu'elle soit enfin étudiée pour ellemême, et non pas en tant que simple outil facilitant d'autres apprentissages - alors même que celles-ci sont menacées par «le monde de l'image » (375), véhiculée par d'autres supports. La « réconciliation entre théoriciens, critiques et pédagogues » (373) que le directeur du numéro appelle de ses vœux, trouve déjà une belle illustration dans un volume qui analyse son objet de manières diverses, mais conjuguées et déclinées selon une intention unique: celle de rendre à la bande dessinée une place qui lui revient de droit dans le cadre d'une étude réévaluée des Humanités, dans un monde où celles-ci oscillent trop souvent entre indifférence et hostilité. 


\section{NOTES}

1. Le Fureteur. Spirou, $8^{\mathrm{e}}$ année, no. 914, 20 octobre 1955.

\section{AUTEURS}

VITTORIO FRIGERIO

Dalhousie University 